\title{
Location of the gene for 21-hydroxylase deficiency
}

\author{
V PUCHOLT, $*$ J S FITZSIMMONS, * K GELSTHORPE, $†$ \\ M A REYNOLDS, + AND R D G MILNER+ \\ From * the Genetic Counselling Service, Nottingham City Hospital, Nottingham; \\ $\dagger$ the Regional Blood Transfusion Service, Longley Lane, Sheffield; and $\ddagger$ the Department of \\ Paediatrics, University of Sheffield, Sheffield
}

SUMMARY HLA typing of 33 families with one or more children suffering from congenital adrenal hyperplasia confirmed that the gene for 21-hydroxylase deficiency is closely associated with the HLA region. Analysis of two families in which recombination of chromosome 6 had occurred indicated that the gene locus is between the $A$ and $D$ loci of the HLA region. The rare allele $B w 47$ was observed in 18 parents and was always associated with the carrier state for 21 -hydroxylase deficiency.

Congenital adrenal hyperplasia $(\mathrm{CAH})$ is a recessively inherited condition most commonly the result of a deficiency of 21-hydroxylase. ${ }^{1}$ There is evidence suggesting that the gene locus for 21-hydroxylase deficiency may be situated on the short arm of chromosome 6 close to the HLA region. ${ }^{2}$ Further work indicates that the gene lies between the $H L A$ locus and the glyoxylate locus, which is the nearest identifiable locus between the HLA region and the centromere. ${ }^{3}$ From the present study it would appear that the gene for 21-hydroxylase deficiency lies within the HLA region and its position is likely to be close to the $H L A-B$ locus.

\section{Patients and methods}

Thirty-three families with one or more children suffering from $\mathrm{CAH}$ have been studied by measurement of HLA types. The families resided in South Yorkshire, Nottinghamshire, and Leicestershire. In nine families there were two affected children, 11 families had two or more children one of whom was affected, and in 13 families there was a single affected child. In total there were 69 children of whom 42 had CAH. There were 24 affected girls, 18 affected boys, 17 normal girls, and 10 normal boys.

Tissue typing was carried out at the Sheffield Regional Blood Transfusion Centre. A standard two stage microlymphocytotoxic test with 120 well characterised typing sera was used to define HLA-A, $-\mathrm{B}$, and $-\mathrm{C}$ antigens. The antigen $\mathrm{Bw} 47$ was identified by the operationally monospecific serum CAS 12551-8W 1160. HLA-DR typing was performed by a two stage microlymphocytotoxic test on B cell Received for publication 15 February 1980 enriched lymphocyte suspensions, $\mathrm{T}$ cell depletion having been obtained by rosetting with sheep red blood cells and separation on Ficoll Triosil. Sixty sera, including several characterised in the 7th and 8th International Histocompatibility Workshops, were used to define the DRw antigens, DRw1 to DRw8.

\section{Results}

The haplotypes of all families studied (tables 1, 2, 3) agreed with the original observation of Dupont et $a l^{2}$ and this has subsequently been confirmed by other workers. ${ }^{4-7}$ In any family the affected children carried identical haplotypes, but the HLA alleles forming the haplotypes differed between families.

In two families recombination of chromosome 6 occurred in the HLA region. In the first family the father had died, but it was possible to reconstruct his probable haplotypes from those of his parents, children, and sister (fig 1). The elder affected daughter carried haplotype $a$ from her father and $c$ from her mother. The second affected daughter carried haplotype $c$ from her mother, but the haplotype from her father suggested that recombination had occurred between the loci $C$ and $A$ of the paternal haplotypes $a$ and $b$. As this girl has CAH it would appear that the locus for 21-hydroxylase deficiency is situated on the HLA segment carrying the $C, B$, and $D$ loci.

In the second recombinant family there were also two affected daughters (fig 2). The elder daughter carried haplotypes $a$ from her father and $c$ from her mother. The younger daughter carried haplotype $a$ from her father. The haplotype from her mother 
TABLE 1 Families with two affected children

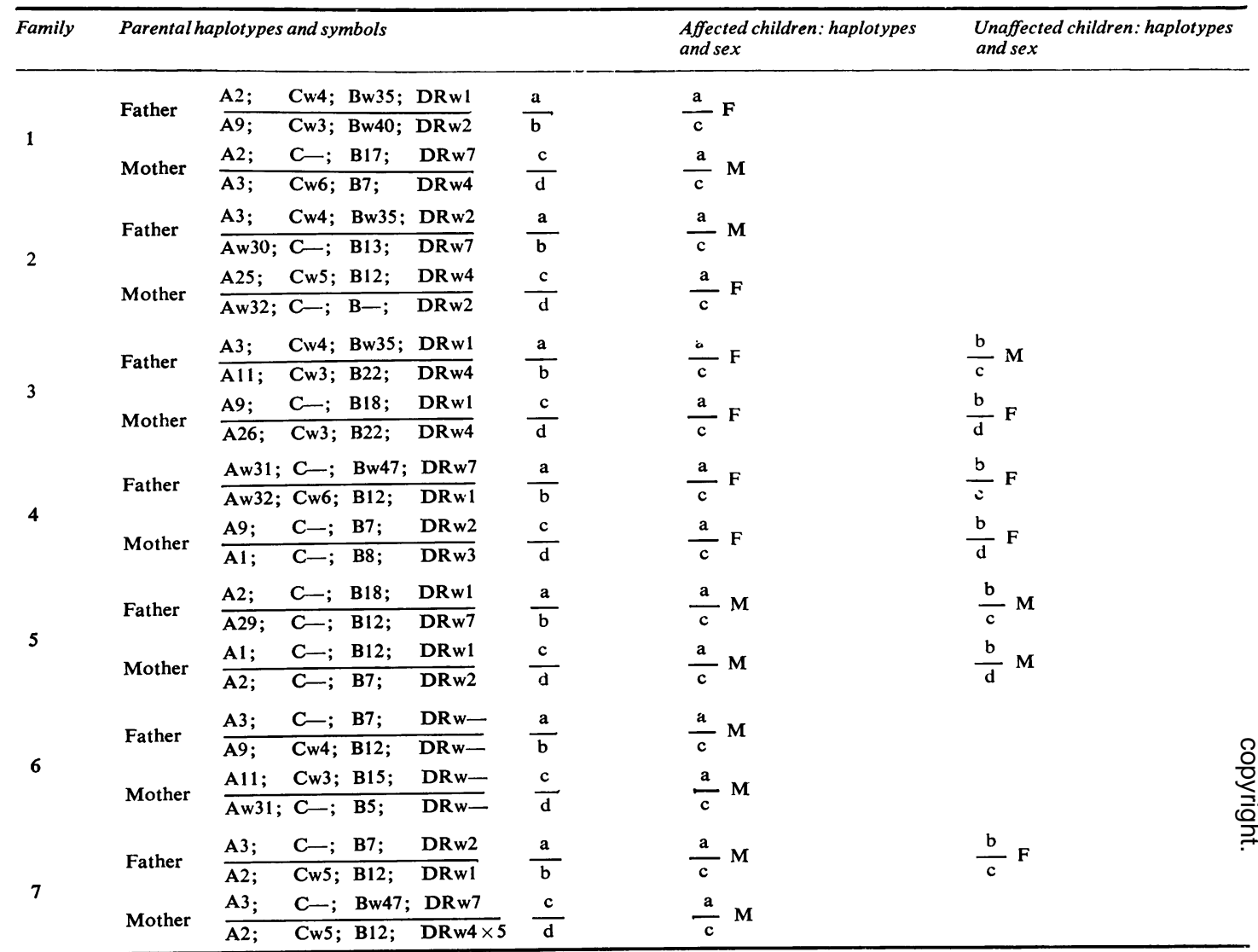

TABLE 2 Families with a single affected child

\begin{tabular}{|c|c|c|c|c|c|c|c|c|c|c|}
\hline Family & Parental & aplotypes & sand sy & vmbols & & & $\begin{array}{l}\text { Affected children: haplotypes } \\
\text { and sex }\end{array}$ & $\begin{array}{l}\text { Unaffect } \\
\text { and sex }\end{array}$ & d children & : haplotypes \\
\hline \multirow[b]{2}{*}{8} & Father & $\frac{\mathrm{A} 3 ;}{\mathrm{Aw} 32 ;}$ & $\begin{array}{l}\mathrm{C}-\text {; } \\
\mathrm{Cw} 4 ;\end{array}$ & $\begin{array}{l}\text { B39; } \\
\text { Bw35; }\end{array}$ & $\frac{\mathrm{DRw} 4}{\mathrm{DRw} 4}$ & $\frac{\mathrm{a}}{\mathrm{b}}$ & $\frac{\mathrm{a}}{\mathrm{c}} \mathrm{F}$ & $\frac{a}{d} F$ & & \\
\hline & Mother & $\frac{\text { A1; }}{A 2 ;}$ & $\begin{array}{l}\mathrm{Cw} 1 ; \\
\mathrm{Cw}_{\mathrm{w}} 3 ;\end{array}$ & $\begin{array}{l}\text { IM2; } \\
\text { B40; }\end{array}$ & $\frac{\text { DRw1 }}{\text { DRw6 }}$ & $\frac{c}{d}$ & & $\frac{b}{d} M$ & & \\
\hline \multirow{3}{*}{9} & Father & Aw33; & $\mathrm{C}-; \mathrm{B}$ & 314; & DRw1 & $\mathbf{a}$ & & b & & \\
\hline & & A11; & Cw4; & B12; & DRw4 & $\bar{b}$ & $\bar{c}^{M}$ & $\bar{d}$ & $\bar{d}$ & \\
\hline & Mother & $\frac{\text { A29; }}{A 3}$ & $\begin{array}{l}\mathrm{C}-; \\
\mathrm{Cw} 3 ;\end{array}$ & $\begin{array}{l}\text { B12; } \\
\text { B15; }\end{array}$ & $\frac{\mathrm{DRw1}}{\mathrm{DRw6}}$ & $\frac{c}{d}$ & & $\frac{a}{d} M$ & $\frac{b}{d} M$ & $\frac{a}{d} F$ \\
\hline \multirow{3}{*}{10} & Father & A26; & Cw6; & B12; & DRw7 & $\mathbf{a}$ & a $F$ & b & a & \\
\hline & Fatner & $\overline{\mathrm{A} 1 ;}$ & $\mathrm{C}-;$ & B17; & $\overline{\text { DRw7 }}$ & $\bar{b}$ & $\bar{c}^{1}$ & $\bar{d}^{N}$ & $\bar{d}^{101}$ & \\
\hline & Mother & $\frac{\mathrm{A} 3 ;}{\mathrm{A} 1:}$ & C-; & B7; & DRw7 & c & & $\frac{\mathrm{b}}{\mathrm{d}} \mathrm{F}$ & & \\
\hline \multirow{4}{*}{11} & & A2; & C-; & B7; & $\begin{array}{l}\text { DRw7 } \\
\text { DRw5 }\end{array}$ & $\mathbf{a}$ & & & & \\
\hline & Father & $\overline{\mathrm{A} 3 ;}$ & $\mathrm{C}-;$ & B7; & $\overline{\mathrm{DRw} 2}$ & $\bar{b}$ & $\bar{c}$ & $\bar{c}^{F}$ & & \\
\hline & & A3; & C-; & Bw47; & DRw7 & c & & b & & \\
\hline & Mother & $\overline{\mathrm{A} 11 ;}$ & $\mathrm{C}-;$ & B40; & $\overline{\text { DRw3 }}$ & $\bar{d}$ & & $\bar{d}^{F}$ & & \\
\hline
\end{tabular}


TABLE 2-continued

\begin{tabular}{|c|c|c|c|c|c|c|c|c|c|c|}
\hline Family & Parental & plotypes & and sym & mbols & & & $\begin{array}{l}\text { Affect } \\
\text { and se. }\end{array}$ & $\begin{array}{l}\text { ed children: haplotypes } \\
x\end{array}$ & $\begin{array}{l}\text { Unat } \\
\text { and }\end{array}$ & $\begin{array}{l}\text { fected children: haplotypes } \\
\text { ex }\end{array}$ \\
\hline \multirow{4}{*}{12} & \multirow{3}{*}{ Father } & A26; & Cw6; & Bw36; & DRw2 & $\mathrm{a}$ & $\mathrm{a}$ & \multirow{4}{*}{$\mathbf{M}$} & $\mathrm{b}$ & \multirow{4}{*}{$-F$} \\
\hline & & $\overline{A 1}$ & $\mathrm{C}-;$ & B7; & $\overline{D R w 2}$ & $\bar{b}$ & $\mathrm{c}$ & & $d$ & \\
\hline & & A2; & Cw5; & B12; & DRw4 & c & & & & \\
\hline & Mother & A1; & $\mathrm{C}-$; & B8; & DRw3 & $\bar{d}$ & & & & \\
\hline \multirow{4}{*}{13} & \multirow{2}{*}{ Father } & A3; & $\mathrm{C}-$; & B7; & DRw4 & a & a & \multirow{4}{*}{$F$} & b & \multirow{4}{*}{$\mathrm{F}$} \\
\hline & & $\overline{\mathrm{A} 1}$ & Cw6; & Bw50; & $\overline{D R w 7}$ & $\mathrm{~b}$ & $\bar{c}$ & & $\bar{d}$ & \\
\hline & \multirow{2}{*}{ Mother } & A2; & $\mathrm{C}-$; & B12; & DRw4 & c & & & & \\
\hline & & $\overline{\mathrm{A} 1}$ & $\mathrm{C}-;$ & B8; & $\overline{D R w 3}$ & $\mathrm{~d}$ & & & & \\
\hline \multirow{4}{*}{14} & \multirow{2}{*}{ Father } & A3; & Cw4; & Bw35; & DRw1 & $\mathbf{a}$ & $\mathbf{a}$ & \multirow{4}{*}{$\mathbf{M}$} & b & \multirow{4}{*}{$F$} \\
\hline & & $\overline{\text { A9; }}$ & Cw3; & Bw22; & $\overline{\mathrm{DRw} 2}$ & $\overline{\mathrm{b}}$ & c & & c & \\
\hline & \multirow{2}{*}{ Mother } & A29; & $\mathrm{C}-;$ & Bw47; & DRw7 & c & & & & \\
\hline & & $\overline{\mathrm{A} 2}$ & Cw6; & B12; & $\overline{D R w 1}$ & $\bar{d}$ & & & & \\
\hline \multirow{4}{*}{15} & \multirow{2}{*}{ Father } & A3; & Cw4; & Bw35; & DRw4 & $\mathrm{a}$ & a & \multirow{4}{*}{$\mathrm{F}$} & $\mathbf{a}$ & M \\
\hline & & A11; & IM2; & Cw1; & $\overline{D R w 5}$ & $\bar{b}$ & $\bar{c}$ & & 4 & 10 \\
\hline & Mother & Al; & $\mathrm{C}-$; & B8; & DRw3 & $\mathrm{c}$ & & & & \\
\hline & मुण & A3; & Cw3; & B40; & $\overline{D R w 2}$ & $\bar{d}$ & & & & \\
\hline & Father & Aw31; & C- & Bw47; & DRw7 & $\mathbf{a}$ & $\mathrm{a}$ & $F$ & b & $F$ \\
\hline 16 & & Aw32; & $\mathrm{C}-$ & Bw44; & $\overline{D R w 1}$ & $\bar{b}$ & c & $\mathbf{r}$ & $\bar{d}$ & $r$ \\
\hline & Mother & Aw24; & $\mathrm{C}$-; & B7; & DRw2 & $\mathrm{c}$ & & & b & $F$ \\
\hline & & $\overline{\mathrm{A} 1}$; & $\mathrm{C}-;$ & B8; & $\overline{D R w 3}$ & $\overline{\mathrm{d}}$ & & & c & $r$ \\
\hline & Father & A2; & Cw6; & Bw47; & DRw7 & $\mathbf{a}$ & a & $F$ & $b$ & $F$ \\
\hline 17 & & A2; & $\mathrm{C}-$; & Bw44; & DRw4 $\times 7$ & $\bar{b}$ & $=$ & $r$ & d & $\mathbf{r}$ \\
\hline & Mother & A3; & Cw6; & Bw47; & DRw7 & c & & & & \\
\hline & & A1; & C-; & B8; & $\overline{D R w 3}$ & d & & & & \\
\hline & Father & A3; & $\mathrm{C}-$; & B7; & DRw- & $\mathbf{a}$ & $\bar{i}$ & $F$ & $\mathrm{~b}$ & $\mathbf{F}$ \\
\hline 18 & & A25; & C-; & B7; & DRw- & $\bar{b}$ & $=$ & & $\dot{\Delta}$ & 1 \\
\hline & Mother & A2; & C—; & B18; & DRw- & $\mathrm{c}$ & . & & & \\
\hline & & $\begin{array}{l}\text { A11; } \\
\text { A3; }\end{array}$ & Cw6; & B17; & DRw- & d & & & & \\
\hline 21 & Father & $\frac{\text { A3; }}{\text { Aw31; }}$ & $\mathrm{C}$-; & Bw47; & DRw7 & $\mathbf{a}$ & & & & \\
\hline 21 & Mother & $\begin{array}{l}\text { Aw31; } \\
\text { A3; }\end{array}$ & $\mathrm{C}-$ & Bw38; & DRw6 & b & $\mathrm{a}$ & $F$ & & \\
\hline & Mother & $\frac{\mathrm{A} 3}{\mathrm{~A} 3}$ & $\mathrm{C}-;$ & Bw47; & DRw7 & c & c & & & \\
\hline & Father & $\begin{array}{l}\text { A3 } \\
\text { A29; }\end{array}$ & C- & B7; & DRw7 & d & & & & \\
\hline 22 & ratner & A9; & $\mathrm{C}-$; & $\begin{array}{l}\text { B12; } \\
\text { B7; }\end{array}$ & $\frac{D R w 1}{D R w 2}$ & $\frac{a}{b}$ & $a$ & & & \\
\hline & Mother & A3; & $\mathrm{C}-$; & Bw47; & DRw7 & c & $\frac{\pi}{5}$ & $\mathrm{~F}$ & & \\
\hline & 1980 & $\overline{\mathrm{A} 9}$ & $\mathrm{C}-$; & B12; & DRw- & $\overline{\mathrm{d}}$ & & & & \\
\hline & Father & A2; & $\mathrm{C}-$; & B5; & DRw5 & a & & & & \\
\hline 23 & & $\mathrm{~A} 2$ & Cw4; & B12; & $\overline{D R w 2}$ & $\bar{b}$ & $a$ & $\mathrm{~F}$ & & \\
\hline & Mother & A28; & C-; & B12; & DRw2 & c & c & 1 & & \\
\hline & & A1; & $\mathrm{C}-$; & B8; & DRw3 & $\mathrm{d}$ & & & & \\
\hline & Father & A3; & $\mathrm{C}-$; & Bw47; & DRw7 & $\mathbf{a}$ & & & & \\
\hline 24 & & A3; & C-; & B7; & DRw7 & $\mathrm{b}$ & a & $M$ & & \\
\hline & Mother & A3; & C-; & Bw47; & DRw7 & c & $c$ & $10 \mathrm{x}$ & & \\
\hline & & A9; & C-; & B5; & DRw7 & d & & & & \\
\hline & Father & A1; & C-; & B8; & DRw- & $\mathbf{a}$ & & & & \\
\hline 25 & & A2; & C-; & B7; & DRw- & $b$ & $\mathbf{a}$ & $M$ & & \\
\hline & Mother & A3; & C-; & Bw47; & DRw- & c & c & Non & & \\
\hline & & Aw36; & $\mathrm{C}-$; & Bw45; & DRw- & $\mathrm{d}$ & & & & \\
\hline & Father & A9; & $\mathrm{C}-$; & B7; & DR w4 & a & & & & \\
\hline 26 & & A2; & C-; & B8; & DRw3 & $b$ & $\mathrm{a}$ & $M$ & & \\
\hline & Mother & A29; & C-; & B12; & DRw1 & c & c & & & \\
\hline & & Aw32; & Cw6; & B14; & DRw6 & $\mathrm{d}$ & & & & \\
\hline
\end{tabular}


TABLE 2-continued

\begin{tabular}{|c|c|c|c|c|c|c|c|c|}
\hline Family & Parental & aplotyp & and sy & mbols & & & $\begin{array}{l}\text { Affected children: haplotypes } \\
\text { and sex }\end{array}$ & $\begin{array}{l}\text { Unaffected children: haplotypes } \\
\text { and sex }\end{array}$ \\
\hline \multirow{4}{*}{27} & \multirow{2}{*}{ Father } & A29; & $\mathrm{C}-$; & B12; & DRw- & $\mathbf{a}$ & \multirow{4}{*}{$\frac{\mathrm{a}}{\mathrm{c}} \mathrm{M}$} & \\
\hline & & $\overline{\mathrm{A} 1 ;}$ & $\mathrm{C}-$; & B8; & DRw- & $\mathrm{b}$ & & \\
\hline & \multirow{2}{*}{ Mother } & A3; & $\mathrm{C}-$; & Bw47; & DRw- & c & & \\
\hline & & A2; & Cw3; & Bw22; & $\overline{\text { DRw- }}$ & $\overline{\mathrm{d}}$ & & \\
\hline \multirow{3}{*}{28} & Father & A2; & $\mathrm{C}-$; & B27; & $\overline{\text { DRw- }}$ & $\bar{b}$ & \multirow{3}{*}{$-\mathbf{M}$} & \\
\hline & \multirow{2}{*}{ Mother } & Al; & $\mathrm{C}-$; & B8; & DRw- & c & & \\
\hline & & A9; & $\mathrm{C}-;$ & B-; & $\overline{\mathrm{DRw}}$ & $\bar{d}$ & & \\
\hline \multirow{3}{*}{29} & \multirow{2}{*}{ Father } & A2; & $\mathrm{C}-$; & Bw50; & DRw I & $\mathbf{a}$ & \multirow{3}{*}{$F$} & \\
\hline & & A29; & $\mathrm{C}-$; & B12; & DRw7 & $\mathrm{b}$ & & \\
\hline & Mother & A2; & Cw6; & B17; & DRw7 & c & & \\
\hline
\end{tabular}

TABLE 3 Familes with a single affected child

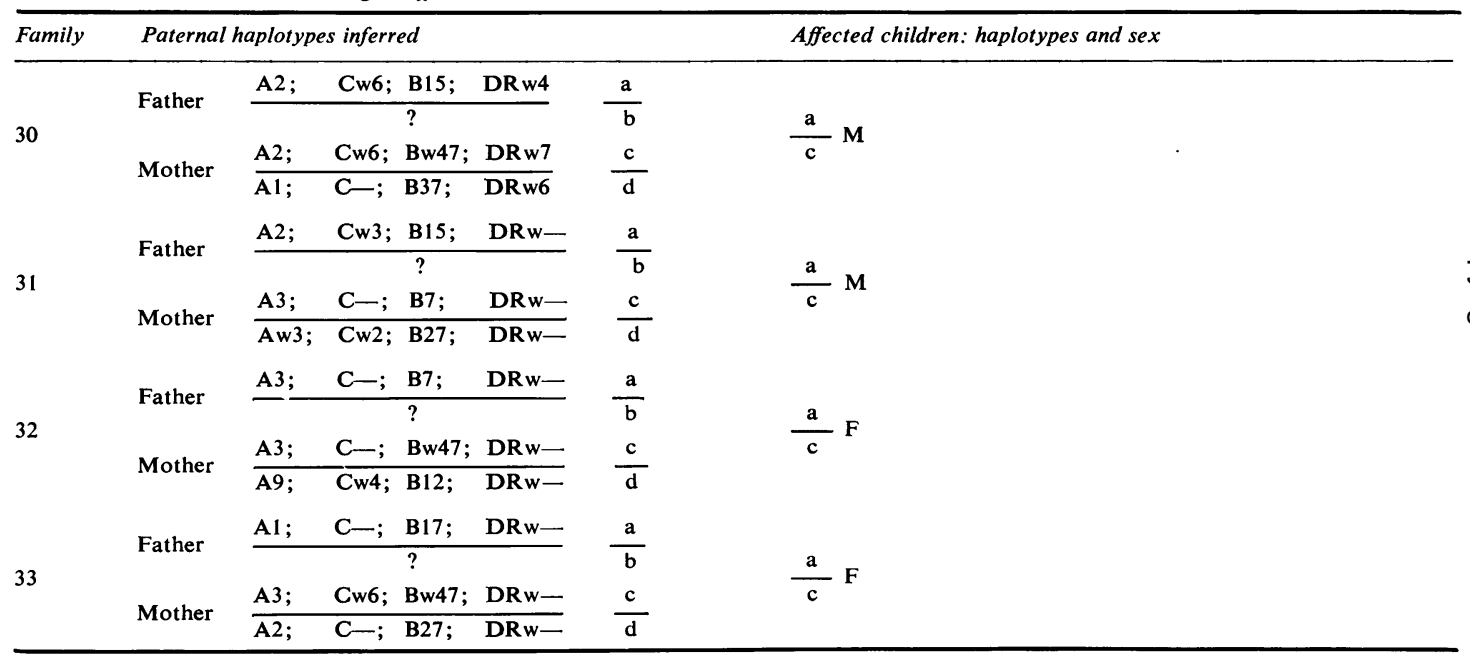

indicated that recombination had occurred between the $B$ and $D R$ loci of the two maternal haplotypes $c$ and $\boldsymbol{d}$. As a confirmatory test of this recombination, a mixed lymphocyte reaction (MLR) test was carried out between the two affected children. HLA-A, B, and $C$ identical sibs are almost invariably nonstimulatory in MLR test. If, however, a different $D / D R$ locus has been substituted in the proposed recombination, that is $D R w 5$ for $D R w I$ in the second daughter, stimulation might be expected to occur. The result of MLR test suggests that the two affected sisters do differ in their $D R$ loci (table 4). The locus for 21-hydroxylase deficiency would therefore appear to be situated in the HLA segment carrying the $A, C$, and $B$ loci. The results from these
TABLE $4 M L R$ in $H L A-B / D R$ recombinant sibs

\begin{tabular}{|c|c|c|c|}
\hline \multirow[t]{2}{*}{ Stimulator cells } & \multicolumn{3}{|c|}{ Responder cells } \\
\hline & $\begin{array}{l}\text { Sib } 2 \\
\text { (DRw 5/5 } \\
\text { recombinant) }\end{array}$ & $\begin{array}{l}\text { Sib } 1 \\
\text { DRw } 1 / 5\end{array}$ & $\begin{array}{l}\text { Unrelated } \\
D R w 2 / 3\end{array}$ \\
\hline $\begin{array}{l}\text { Sib } 2 \text { 'm' } \\
\text { DRw } 5 / 5\end{array}$ & - & 1 & 52 \\
\hline $\begin{array}{l}\text { Sib } 1 \text { 'm' } \\
\text { DRw } 1 / 5\end{array}$ & 37 & 一 & 42 \\
\hline $\begin{array}{l}\text { Unrelated ' } m \text { ' } \\
\text { DRw } 2 / 3\end{array}$ & 60 & 45 & - \\
\hline
\end{tabular}

' $m$ ', mytocin treated cells.

Cells from the second daughter (recombinant DRw5/DRw5) are stimulated by cells from the first daughter (DRw5/DRw1). Predictably, cells from the first daughter (DRw1/DRw5) are not stimulated by the cells from her recombinant sister (DRw5/DRw5). Results of the MLR test are expressed as relative responses. 


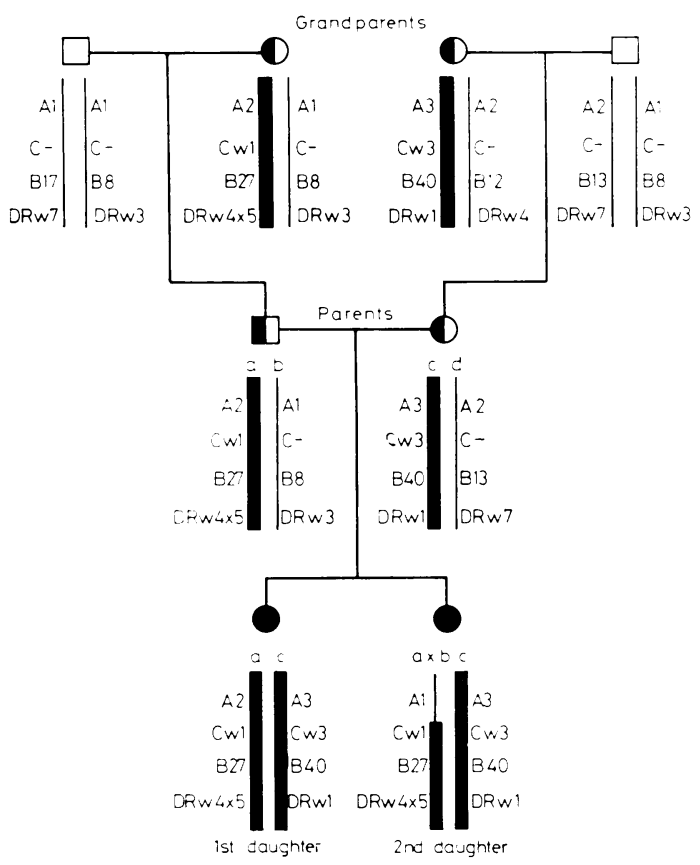

Ha 1 Haplotypes of family l. Both daughters are affected. The second daughter carries evidence of recombination between loci $A$ and $C$ of the paternal haplotypes $(a ; b)$. (Haplotypes of the father are inferred from those of his relatives.) The thick line represents the chromosome carrying the gene locus responsible for 2I-hydroxylase deficiency.

two families indicate that the 21-hydroxylase gene probably lies in the HLA region between the $A$ and $D$ loci.

In our earlier report ${ }^{8}$ we noted an unusually frequent association of a rare HLA allele, $B w 47$, with the gene responsible for 21-hydroxylase deficiency. In the present series Bw47 was observed in the haplotypes of 18 parents and was also present in the haplotype of all their affected children.

\section{Discussion}

The results of this study agree with and extend earlier reports that the gene for 21-hydroxylase deficiency lies within the HLA region. ${ }^{3}$ The practical implications of this observation are important. Experience from the genetic counselling clinic in Nottingham and elsewhere indicates that many parents with one affected child would welcome a dependable prenatal diagnostic test for this condition in later pregnancies. Recent reports have described

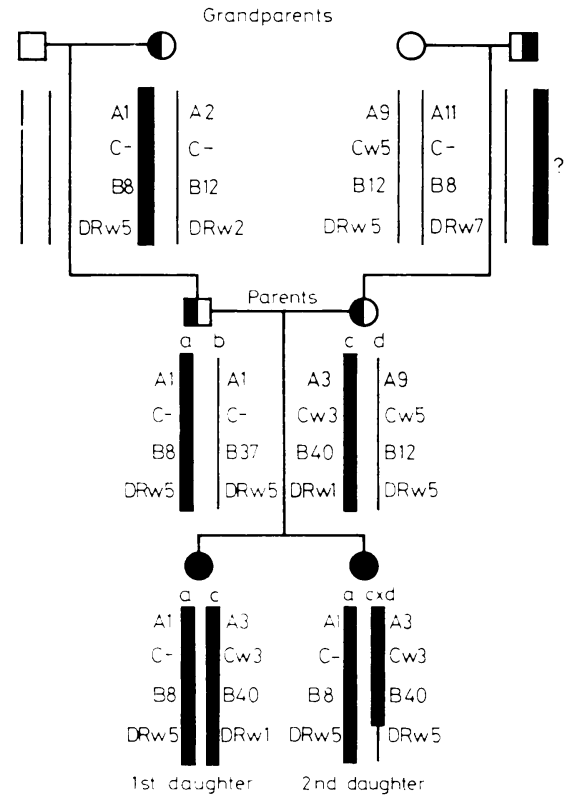

Fla 2 Haplotypes of family 2. Both daughters are affected. The second danghter carries evidence of recombination between $B$ and $D R$ loci of the maternal haplotypes $(c ; d)$. The thick line represents the chromosome carrying the gene locus responsible for 21-hydioxylase deficiency. (It is not known if the maternal grandfather was heterozyous for the gene responsible.)

two methods of diagnosing 21-hydroxylase deficiency prenatally. One method uses tissue typing of fetal cells." ${ }^{10}$ The other method measures the concentration of $17 \%$-hydroxyprogesterone in the amniotic fluid which is considerably raised if the fetus is affected. ${ }^{11}$ Both tests can be carried out on the same sample of amniotic fluid obtained in the second trimester of pregnancy. If confirmcd the two tests would make the prenatal diagnosis more reliable.

In addition, HLA typing among relatives of an affected subject may enable heterozygotes to be identified. This could be of importance in countries where the incidence of 21-hydroxylase deficiency is high, for example, Wales and Switzerland.

We are grateful to all those consultant paediatricians and physicians in the Trent Region who permitted us to study their patients and their relatives.

\section{References}

1 Zurbrugg RP. In: Gardner LI. ed. Endocrine and genetic discease's of childthood and adolescence. Philadelphia: Saunders, 1969:407-24. 
2 Dupont B, Smithwick EM, Oberfield SE, Lee TD, Levine LS. Close genetic linkage between HLA and congenital adrenal hyperplasia. Lancet $1977 ;$ ii:1309-11.

3 Levine LS, Zachmann M, New MI, et al. Genetic mapping of the 21-hydroxylase deficiency gene within the HLA linkage group. $N$ Engl J Med 1978;299:911-5.

4 Price DA, Klouda PT, Harris R. HLA and congenital adrenal hyperplasia. Letter. Lancet 1978;i:930-1.

5 Weitkamp LR, Bryson M, Bacon GE. HLA and congenital adrenal hyperplasia. Letter. Lancet 1978;i:931-2.

6 Zappacosta S, De Felice M, Minozzi M, Lombardi G, Valentino R, Vanacore G. HLA and congenital adrenal hyperplasia. Letter. Lancet 1978 ;ii:524.

7 Murtaza LM, Hughes IA, Sibert JR, Balfour IC. HLA and congenital adrenal hyperplasia. Letter. Lancet $1978 ; 2: 524$.

8 Pucholt V, Fitzsimmons JS, Gelsthorpe K, Pratt RF,
Doughty RW. HLA and congenital adrenal hyperplasia. Letter. Lancet 1978 ;ii:1046.

9 Pollack MS, Levine LS, Pang S, et al. Prenatal diagnosis of congenital adrenal hyperplasia (21-hydroxylase deficiency) by HLA-typing. Lancet 1979; i:1107-9.

10 Couillin P, Nicholas N, Boué J, Boué A. HLA typing of amniotic fluid cells applied to prenatal diagnosis of congenital adrenal hyperplasia. Letter. Lancet 1979;i: 1076.

11 Hughes IA, Laurence KM. Antenatal diagnosis of congenital adrenal hyperplasia. Lancet 1979 ;ii :7-9.

Requests for reprints to $\mathrm{Dr} \mathbf{J} \mathrm{S}$ Fitzsimmons, Trent Sub-Regional Genetic Counselling Service, Nottingham City Hospital, Hucknall Road, Nottingham NG5 1PB. 